\title{
Suboptimal Use of Neural Information in a Mammalian Auditory System
}

\author{
Laurel H. Carney, ${ }^{1}$ Muhammad S. A. Zilany, ${ }^{1}$ Nicholas J. Huang, ${ }^{1}$ Kristina S. Abrams, ${ }^{1}$ and Fabio Idrobo ${ }^{2}$ \\ ${ }^{1}$ Departments of Biomedical Engineering, and Neuroscience and Anatomy, University of Rochester, Rochester, New York 14642 , and ${ }^{2}$ Department of \\ Psychology, Boston University, Boston, Massachusetts 02215
}

Establishing neural determinants of psychophysical performance requires both behavioral and neurophysiological metrics amenable to correlative analyses. It is often assumed that organisms use neural information optimally, such that any information available in a neural code that could improve behavioral performance is used. Studies have shown that detection of amplitudemodulated (AM) auditory tones by humans is correlated to neural synchrony thresholds, as recorded in rabbit at the level of the inferior colliculus, the first level of the ascending auditory pathway where neurons are tuned to AM stimuli. Behavioral thresholds in rabbit, however, are $\sim 10 \mathrm{~dB}$ higher (i.e., 3 times less sensitive) than in humans, and are better correlated to rate-based than temporal coding schemes in the auditory midbrain. The behavioral and physiological results shown here illustrate an unexpected, suboptimal utilization of available neural information that could provide new insights into the mechanisms that link neuronal function to behavior.

\section{Introduction}

Considerable effort in systems-level neuroscience research focuses on the relation between neural responses and behavior. Optimal neural coding strategies are often assumed, and several theoretical and neurophysiological studies have examined optimal schemes for coding and processing in sensory systems using information theory (Borst and Theunissen, 1999; Barlow, 2001; Laurent, 2002; Harper and McAlpine, 2004; Knill and Pouget, 2004; Lu and Wang, 2004; Smith and Lewicki, 2006; Clemens et al., 2011). However, when both behavioral and physiological datasets are available for the same species, neural correlates of behavioral thresholds can be directly explored, and hypotheses concerning aspects of the neural responses that code different qualities of sensory stimuli can be formally tested. This approach is applied here to the coding of amplitude modulations (AM) of acoustic stimuli.

Sensitivity to AM has been investigated extensively in humans because fluctuations in amplitude represent an important form of temporal information in speech, music, and environmental sounds (Rosen, 1992; Rieke et al., 1995; Attias and Schreiner,

Received July 17, 2013; revised Nov. 6, 2013; accepted Dec. 15, 2013.

Author contributions: L.H.C. and F.I. designed research; L.H.C., M.S.A.Z., N.J.H., and K.S.A. performed research; L.H.C., M.S.A.Z., N.J.H., K.S.A., and F.I. analyzed data; L.H.C. and F.I. wrote the paper.

This work was supported by US National Institutes of Health Grant R01-DC001641 (L.H.C.) from the National Institute on Deafness and Other Communication Disorders. We thank D. Cameron, T. Li, and Y. Gai for helpful comments on the paper, D. Schwarz for assistance with analysis and figures, and J. M. Harrison for assistance with early AM-detection experiments in rabbit.

The authors declare no competing financial interests.

Correspondence should be addressed to Laurel H. Carney, Department of Biomedical Engineering, University of Rochester, 601 Elmwood Avenue, Box 603, Rochester, NY 14642. E-mail: Laurel.Carney@Rochester.edu.

M. Zilany's present address: Department of Biomedical Engineering, University of Malaya, Kuala Lumpur, Malaysia.

N. Huang's present address: Department of Biomedical Engineering, Johns Hopkins University, Baltimore, MD. DOI:10.1523/JNEUROSCI.3031-13.2014

Copyright $\odot 2014$ the authors $\quad 0270-6474 / 14 / 341306-08 \$ 15.00 / 0$
1997; Nelken et al., 1999; Smith et al., 2002; Elliott and Theunissen, 2009). AM also plays a critical role in detection of stimuli in the presence of noise (Richards, 1992; van der Heijden and Kohlrausch, 1995; Davidson et al., 2009a,b; Jørgensen and Dau, 2011; Mao et al., 2013).

The inferior colliculus (IC) is a key location for studying AM coding because it is the first location in the ascending pathway where rates are well tuned to AM frequency. Physiological studies of AM have typically used 100\% modulated stimuli (Langner and Schreiner, 1988; Joris and Yin, 1998; Krishna and Semple, 2000; Liang et al., 2002; Joris et al., 2004; Lu and Wang, 2004; Bendor and Wang, 2010; Rabang et al., 2012). Modulation depths of interest for the comparison of neural and perceptual thresholds, however, are much smaller, on the order of -25 to $-30 \mathrm{~dB}$ relative to full modulation (i.e., $20 \log _{10}(m)$, where $m$ is the modulation index), or $\sim 3-6 \%$ modulation depth (Kohlrausch et al., 2000). Neural thresholds for detection of AM can be estimated as the lowest modulation depth that causes a change in discharge rate or significant synchrony to the stimulus envelope (Nelson and Carney, 2007; Rosen et al., 2010). Synchrony of responses in the IC to AM stimuli often emerges at lower modulation depths than changes in rate. AM synchrony thresholds of IC in rabbit (Nelson and Carney, 2007) and auditory cortex in primate (Malone et al., 2010) are closer to human behavioral thresholds than are rate-based threshold estimates. These results are consistent with the general assumption that the nervous system uses the best available information, in this case synchrony, for detection of a feature of complex sounds.

A primary caveat for these results is the comparison of behavioral thresholds of human and neural thresholds from different species. Here, rabbit behavioral thresholds for AM tones and noise were compared with neural thresholds in the IC of awake 

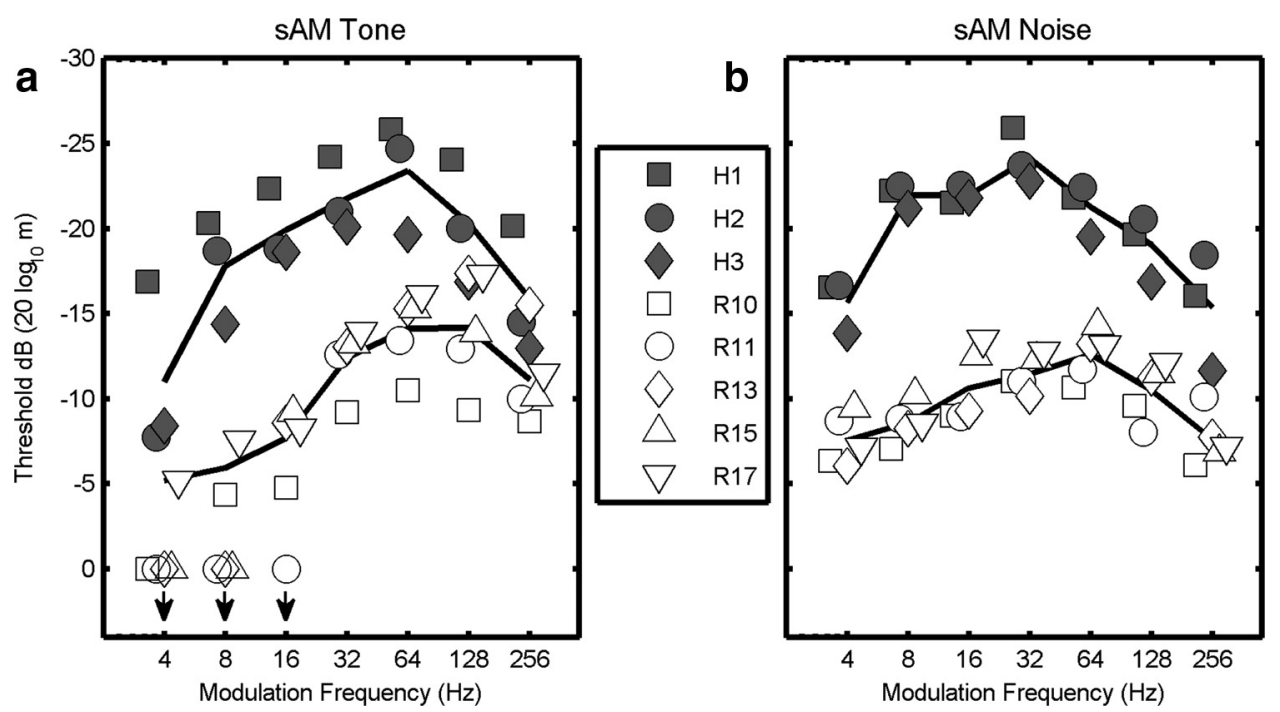

Figure 1. Modulation transfer functions (i.e., sAM detection threshold vs $F_{m}$ ) for human and rabbit. Symbols (solid, human; open, rabbit) represent thresholds determined for individual listeners; solid lines are the mean thresholds for each species. More sensitive detection thresholds are at the top of each graph. $\boldsymbol{a}$, Thresholds for sAM tones. Stimuli were sAM $5 \mathrm{kHz}$ tones presented at $50 \mathrm{~dB}$ SPL. Downward arrows indicate conditions for which the animals could not perform at criterion; mean thresholds do not include these values. $\boldsymbol{b}$, Thresholds for sAM wideband noise presented at 20 dB SPL spectrum level; noise bandwidth was $0.1-10 \mathrm{kHz}$. All thresholds were based on a single-interval choice task with $500 \mathrm{~ms}$ duration stimuli. For all frequencies tested humans almost invariably had higher sensitivity to AM than rabbits.

rabbit. In addition, human thresholds for AM detection were estimated using procedures matched to those for rabbit.

\section{Materials and Methods}

All behavioral and physiological experiments in rabbits were approved by the University Committee on Animal Resources at the University of Rochester. Psychophysical experiments in human listeners were approved by the Research Subjects Review Board at the University of Rochester.

Behavioral methods. Amplitude-modulation detection thresholds of five female Dutch-belted rabbits (aged 0.5-3 years) and three adult human listeners with normal hearing (aged 20-48 years) were tested using operant procedures. Stimuli were either $5 \mathrm{kHz}$ tones presented at $50 \mathrm{~dB}$ sound pressure level (SPL) or wideband $(0.1-10 \mathrm{kHz})$ noise presented at $20 \mathrm{~dB}$ SPL spectrum level ( $\sim 60 \mathrm{~dB}$ SPL root mean squared). Stimuli were sinusoidally amplitude-modulated (sAM) at frequencies ranging from 4 to $256 \mathrm{~Hz}$ in 1 octave steps. Modulation depths, $20 \log _{10}(m)$, ranged from -26 to $0 \mathrm{~dB}$, where $m$ is the modulation depth; $0 \mathrm{~dB}$ modulation depth corresponds to $m=1$, or a $100 \%$ modulated stimulus. All stimuli were $500 \mathrm{~ms}$ in duration and were gated on and off with $50 \mathrm{~ms}$ duration raised-cosine ramps.

Rabbits were trained and tested in an operant two-alternative nonforced choice task, using apparatus and procedures previously described (Gai et al., 2007), except that no audiovisual stimuli were presented after reporting responses here because this feedback was subsequently found not to improve detection thresholds (F. Idrobo, personal communication). Responses were nose-pokes into three horizontally arranged stainless-steel rings; each monitored using an infrared beam. A nosepoke in the center hole served as the observing response and initiated a stimulus; after stimulus presentation, the animal made a reporting response with a nose-poke in the right or left hole, depending upon whether the stimulus was perceived as modulated (50\% of trials) or unmodulated (50\%), respectively. Animals were reinforced for a correct response with a food pellet. An incorrect response was followed by a $5 \mathrm{~s}$ lights-out timeout period; any responses during the timeout period reset the $5 \mathrm{~s}$ timer. Bias in the percentage of responses to the left or right nose-poke hole was monitored and controlled by varying the percentage of multipellet reinforcements to the biased-against side.

Rabbits were initially trained on $100 \%$ modulated stimuli ( $0 \mathrm{~dB}$ modulation depth) until $85 \%$ correct performance was achieved. Secondary training using long (300-400 trial) two-down, one-up tracks (Levitt, 1971) accustomed the animals to lower modulation depths. Training was performed over a month or more for each animal. During subsequent testing modulation depth was varied across trials to estimate detection thresholds using a Bayesian procedure (Kontsevich and Tyler, 1999). This procedure determines the most likely psychometric function, from a large set of precalculated candidate functions, based on the entire set of stimulus-response pairs. During the test, each stimulus is selected to maximize the information that will be gained about the likelihoods of the candidate psychometric functions based on the possible outcomes of each trial. As a result, the stimulus parameter of interest, in this case AM depth, varied widely at the beginning of each test block, but quickly converged to threshold. The behavioral threshold estimate for each test block was the AM depth that corresponded to $70.7 \%$ correct on the psychometric function that was found to be most consistent with the responses for that block. This procedure facilitated the efficient measurement of multiple threshold estimates, each based on a relatively small numbers of trials (e.g., 60 trials), while avoiding the bias in short adaptive tracks introduced by the experimenter's choice of the starting point. Such a bias has been reported for human listeners (Green, 1993) and was observed in initial AM-detection experiments in the rabbit. Each AMdetection threshold estimate was based on $\sim 30$ modulated and 30 unmodulated trials; modulation depth was varied within a test block and modulation frequency $\left(F_{\mathrm{m}}\right)$ was held constant over a series of blocks, until a stable threshold estimate was obtained. Behavioral measurements were repeated if there was any evidence of improvement due to learning or practice effects, until stable performance was established. Final thresholds for each $F_{\mathrm{m}}$ condition were based on 24 unbiased estimates that were stable, i.e., if a statistically significant trend as determined by linear regression was present across the threshold estimates, or if the SD of the threshold estimates exceeded $4 \mathrm{~dB}$, testing was continued. Stimulus conditions were tested in different sequences for different animals; each animal was tested for several weeks using only tones or only noise, and modulation frequencies were varied sequentially (in different directions for different animals) to avoid large changes in stimulus features across adjacent sessions. Each animal was tested in daily sessions over 7-8 months to complete all of the $F_{\mathrm{m}}$ conditions for both sAM tones and noise. The entire set of conditions was tested twice for each animal. If two thresholds, each based on 12 estimates, differed by more than 1 SD, then testing for that condition was repeated. Repeated measurements were most common for the low $F_{\mathrm{m}}$ sAM tone conditions that were difficult for the rabbits (Fig. 1a). Finally, the two sets of 12 estimates were combined into a single threshold based on 24 estimates. 
Stimuli and procedures for human testing were generally matched to those used for the rabbit. A single-interval, two-alternative choice task was used; stimuli were presented diotically via TDH-30 headphones. The subject initiated a stimulus with an observing response, by clicking on a central button with a computer mouse. The reporting response was a click on the button to the left or right to indicate whether the stimulus was perceived as unmodulated (50\% of trials) or modulated (50\%). The Bayesian procedure (Kontsevich and Tyler, 1999) was used to estimate threshold; the range of modulation depths tested for human listeners was $-36 \mathrm{~dB}$ to $-10 \mathrm{~dB}$. Due to higher consistency in human performance, final thresholds for each $F_{\mathrm{m}}$ condition were based on the last eight unbiased estimates. Human and rabbit thresholds are within $\sim 5 \mathrm{~dB}$ of each other at $5 \mathrm{kHz}$, the frequency of the sAM tone carrier (Sivian and White, 1933; Heffner and Masterton, 1980). The difference in audibility of the suprathreshold $5 \mathrm{kHz}$ sAM tone would be expected to result in a 1-2 dB threshold difference across species, based on the level-dependence observed in humans (Kohlrausch et al., 2000), which is similar to that in rabbit (unpublished observations). For sAM noise, AM detection thresholds are not level-dependent in human (Viemeister, 1979) or rabbit (unpublished observation).

Physiological methods. Physiological responses were recorded from neurons in the central nucleus of the IC of three awake female Dutchbelted rabbits using tetrodes made with 12 - or 18 - $\mu \mathrm{m}$-diameter epoxycoated platinum iridium wire. Tetrodes protected by stainless-steel guide tubes were implanted under anesthesia, and the tetrodes were advanced through the IC using a manual microdrive (Neuralynx 5-Drive). Physiological criteria for IC responses were used during testing, and histological verification of electrode tract locations was made after experiments were completed. Data were recorded during 287 daily 2 -h-duration sessions; the responses reported here were based on a total of 378 wellisolated single neurons. Action potentials (spikes) were sorted off-line using a semiautomated procedure based on features of the spike waveforms (Schwarz et al., 2012). Typically, one or more neurons per tetrode remained stable throughout the $2 \mathrm{~h}$ recording session. Neural units were categorized as "single-unit" or "multi-unit" depending upon analyses of the interspike intervals, a spike cluster separation metric, and the quality of the spike waveform histograms (Schwarz et al., 2012). Only results for single units are included in the figures here; however, the results were generally similar for multi-unit recordings. Fewer symbols than 378 appear in Figures 3 and 4 because many neurons did not have statistically significant changes in response to different modulation depths, and thus neural detection thresholds could not be obtained. In keeping with previous studies comparing behavioral and neural thresholds for AM (Rosen et al., 2010; Johnson et al., 2012), only cells that exhibited sensitivity to AM depth were included in the comparisons; inclusion of insensitive cells would, in general, elevate neural threshold estimates, if the responses were pooled across populations of neurons. For sAM tones, rate thresholds were obtained for 75 neurons, and synchrony thresholds were obtained for 93 neurons; for sAM noise, rate thresholds were obtained for 103 neurons and synchrony thresholds for 106 neurons. Comparison of behavioral thresholds with neural thresholds of cells that showed sensitivity for AM is consistent with previous studies if the tetrode position was stable across days; it is possible that on occasion the same neurons were studied in subsequent sessions. Approximately half of the sessions focused on SAM tones and half on SAM wideband noise $(0.1-20 \mathrm{kHz})$. In addition, half of the sessions focused on contralateral stimulation whereas the other half focused on binaural (diotic) sAM stimulation.

Acoustic stimuli were presented from Beyer-Dynamic headphones (DT-48) via custom-made ear molds (Hal-Hen Perform $\mathrm{H} / \mathrm{H}$ material). The tone stimuli had a carrier frequency of $5 \mathrm{kHz}$ for the behavioral studies in both rabbit and human, whereas physiological thresholds were estimated at tone frequencies chosen near the best audio frequencies (BF, the stimulus frequency that elicits the largest response at a given SPL) of the neurons. Noise stimuli for behavioral thresholds had a $10 \mathrm{kHz}$ bandwidth, whereas noise for physiological stimuli had a $20 \mathrm{kHz}$ bandwidth. Otherwise, the parameters of the physiological stimuli (e.g., duration, sound level, modulation frequency, and modulation depth) matched those of the behavioral experiments. Stimuli with different modulation depths were presented in random sequence. Stimulus carrier and modulation frequencies were matched to the $\mathrm{BF}$ and best modulation frequency (BMF) of one or more neurons in the group being recorded during that particular session. All statistically significant neural thresholds were reported, regardless of the detailed match between stimulus and tuning properties, although thresholds would in general be most sensitive when the stimulus properties were aligned with the tuning properties.

Neural detection thresholds for AM stimuli, based on changes in average discharge rate, were estimated with a neurometric strategy using receiver-operating characteristic (ROC) analysis (Egan, 1975). This analysis identifies the modulation depth for which $70.7 \%$ correct AM detection could be made using distributions of average rates in response to unmodulated stimuli and stimuli with different modulation depths. Application of the ROC analysis to the synchrony thresholds is problematic because of the high variance of synchrony metrics when calculated for responses to single trials, or even responses combined across small numbers of trials, depending on response rates. Instead, neural thresholds based on synchronization of action potentials to the stimulus envelope were estimated based on two tests of circular statistics: (1) The synchrony coefficient, or vector strength (Goldberg and Brown, 1969), was computed to assess phase-locking to the sAM envelope; near synchrony threshold, period histograms based on $F_{\mathrm{m}}$ were unimodal. The Rayleigh statistic was used to test for significance of the vector strength. This test statistic was required to exceed $13.8(p<0.001)$ (Mardia and Jupp, 2000). (2) The Kuiper test for significant differences in circular distributions (Kuiper, 1960; $p<0.05$ ) was used to test the hypothesis that a period histogram in response to a modulated stimulus differed significantly from the period histogram in response to the unmodulated stimulus. The latter test was required because reproducible ("frozen") noise waveforms were used for the modulation depth curves in response to sAM noise, and some IC neurons phase-lock strongly to temporal features in the unmodulated (or weakly modulated) noise, which can result in artifactually significant phase-locking to the unmodulated stimulus. Similarly, onset and offset responses, which can elicit strongly timelocked responses, were excluded from the analysis window, which spanned 75-450 ms referenced to stimulus onset $(t=0 \mathrm{~ms})$. The neural synchrony threshold was the lowest modulation depth for which both the Rayleigh and Kuiper tests were satisfied. Neural threshold estimates (Figs. $2 c, 3,4$ ) were based on rate or synchrony measures across 50 stimulus presentations for each modulation depth.

\section{Results}

\section{Behavioral thresholds for AM detection}

Behavioral modulation transfer functions (MTFs) show detection threshold as a function of modulation frequency $\left(F_{\mathrm{m}}\right)$. Thresholds for detection of sAM tones with $5 \mathrm{kHz}$ tone carriers are shown for rabbits ( $n=5$; open symbols) and humans $(n=3$; filled symbols; Fig. 1a). For consistency with the psychophysical literature (Viemeister, 1979), the AM detection thresholds are plotted as $20 \log _{10}(m)$, where $m$ is the modulation index, with values located near the top of each plot indicating lower (more sensitive) detection thresholds. Negative threshold values represent modulations of $<100 \%$, or $m<1$. At all modulation frequencies tested, humans detected much smaller amplitude modulations than rabbits. Note that the human thresholds were measured using a single-interval task matched to that used for the rabbits (see Materials and Methods). Except at very low $F_{\mathrm{m}}$, these thresholds were generally consistent with human thresholds estimated using two- or three-interval tasks typical of psychophysical studies (Viemeister, 1979; Kohlrausch et al., 2000).

Although the rabbits tested here were overtrained in the behavioral tasks, they were consistently and particularly insensitive to amplitude modulations of tone carriers at low $F_{\mathrm{m}}$ (Fig. 1a). Some individual rabbits were unable to detect modulations for $F_{\mathrm{m}}<16 \mathrm{~Hz}$, even for $100 \%$ modulated stimuli (Fig. $1 a$, bottom left, downward arrows). The corresponding slight worsening of 



Figure 2. Response properties of two representative neurons in the IC of awake rabbit. $\boldsymbol{a}$, Response maps. Audio frequency tuning is described as changes in average discharge rate as a function of tone frequency at several sound levels. The gray lines indicate spontaneous rate. $\boldsymbol{b}$, MTFs based on discharge rate. Examples of bandpass (left) and band-reject (right) MTFs are shown. BMF is determined by the peak of bandpass MTFs or the minimum in band-reject MTFs. $c$, Average discharge rate (blue) and synchrony (red, sync coefficient) as a function of modulation depth. Neural thresholds for AM detection for each metric (arrows) were estimated from the responses to stimuli that varied in modulation depth. Responses to unmodulated (U) stimuli are shown by the open symbols at the left. Distributions of the average discharge rate for each modulation depth were analyzed using the ROC technique to determine neural rate thresholds for AM detection. Neural thresholds based on synchrony were computed using circular statistics applied to period histograms binned on the modulation frequency (see Materials and Methods). $m$, Modulation depth.

human thresholds at low $F_{\mathrm{m}}$ was not observed in a two-interval control task, suggesting that at very low modulation frequencies, for which relatively few cycles of the stimulus are presented, a two-interval task is beneficial. Although the one-interval task may have contributed to the reduced sensitivity at low $F_{\mathrm{m}}$, it fails to explain the difference between human and rabbit performance at all modulation frequencies.

Behavioral thresholds for detection of sAM wideband noise stimuli show that, similar to the case for sAM tones presented above, humans were more sensitive than rabbits to AM noise (Fig. 1b, compare open and filled symbols). Changes in threshold at low modulation frequencies were not as dramatic for sAM noise stimuli as for sAM tones. All individuals, human and rabbit, were able to detect modulations at every $F_{\mathrm{m}}$ tested with the sAM noise stimuli. These behavioral results indicate a consistent, species-specific aspect of AM detection, with humans being more sensitive than rabbits.

\section{Neural thresholds for AM detection}

IC neurons are generally tuned to a $\mathrm{BF}$ (Fig. 2a). In addition, the IC is the first level of the ascending pathway where neurons have response rates that are strongly tuned to $F_{\mathrm{m}}$ of AM stimuli (Fig. $2 b$ ).

Each physiological session included determination of response maps for each neuron (discharge rate as a function of stimulus frequency at several sound levels), which were used to identify the neuron's BF and response-map type (Fig. 2a). Responses to AM stimuli can be described by MTFs, which display average discharge rate as a function of $F_{\mathrm{m}}$ (Fig. 2b). The MTF was measured to identify the BMF, the modulation frequency that elicits the highest discharge rate for neurons with bandpass MTFs (Fig. 2b, left) or the lowest rate for cells with band-reject MTFs (Fig. $2 b$, right). MTFs in the IC are predominantly bandpass, low-pass, or band-reject (Müller-Preuss et al., 1994; Krishna and Semple, 2000; Nelson and Carney, 2007). Rate versus modulation depth curves were then measured for sAM wideband noise or tone stimuli, with $F_{\mathrm{m}}$ near BMF (Fig. 2c). Neural thresholds (Fig. 2c, arrows) were estimated from the responses in the modulation depth curves using ROC analyses of rate and statistical analyses of synchronization (Egan, 1975).

Single-unit neural thresholds for sAM detection for responses across a range of $F_{\mathrm{m}}$ were estimated based on discharge rate (Fig. 3a) and synchrony (Fig. 3b). When comparing the neural and behavioral thresholds, a key question is whether the most sensitive neurons (i.e., the symbols closest to the top of the plot) have thresholds that are sufficient to explain the behavioral sensitivity. Mean behavioral thresholds for rabbits (gray line) and humans (black line) $\pm 1 \mathrm{SD}$ (gray regions) are shown for comparison to the neural thresholds (Fig. 3). The most sensitive neural thresholds based on average discharge rate (Fig. 3a, topmost symbols) can explain rabbit behavioral thresholds. All of the rate-based neural thresholds, however, were substantially less sensitive than human behavioral thresholds. Reports of AM tuning in the IC typically suggest that few neurons are tuned to low $F_{\mathrm{m}}$, as is true in these dataset; although human thresholds for low $F_{\mathrm{m}}$ exceed both rate and synchrony thresholds, this may reflect a sampling problem. A recent description of a topographical map for $F_{\mathrm{m}}$ in the IC (Baumann et al., 2011) suggests that the surgical approach might bias the $F_{\mathrm{m}}$ distribution.

In contrast the most sensitive synchrony-based neural thresholds (Fig. 3b) were consistently more sensitive (higher on the plot) than rate-based thresholds, and more sensitive than rabbit behavioral thresholds. The fact that rabbit behavioral thresholds 
are less sensitive than neural thresholds suggests that neural information encoded by IC neurons is not being used by the rabbit. The most sensitive synchronybased neural thresholds were sufficient to explain human behavioral detection thresholds, for both sAM tones and noise.

The data in Figure 3 combines thresholds for monaural and binaural stimulus paradigms, and for different IC response-map types. There were no evident differences in the distribution of neural thresholds based on responses of cells to contralateral or binaural (diotic) stimuli, nor did the thresholds differ for neurons with bandpass, low-pass, or band-reject MTFs, or for neurons with different response-map types. These results indicate that the most sensitive rate thresholds of IC neurons, regardless of response type, are consistent with rabbit behavioral thresholds, whereas the most sensitive synchrony-based thresholds are inconsistent with rabbit behavior yet are sufficient to explain human behavioral thresholds.

The neural thresholds (Fig. 3) are summarized in distributions (Fig. 4) that compare neural and behavioral thresholds combined across $F_{\mathrm{m}}$. The mean rabbit behavioral threshold is centered on the abscissa, as indicated by the vertical dashed line. The distributions of rate thresholds are to the left of the mean behavioral thresholds, with the most sensitive (righthand most) rate thresholds falling within $1 \mathrm{SD}$ of the mean behavioral threshold (gray). The distribution of synchrony thresholds is shifted to the right, with the most sensitive thresholds falling outside the range of behavioral thresholds. These distributions indicate that the best neural rate thresholds are sufficient to explain the rabbit behavioral thresholds, whereas synchrony to the envelope is required to explain the sensitivity to AM observed in human psychophysical thresholds. The fact that the most sensitive information in the responses of rabbit IC neurons to AM sounds is not accessed by the rabbit brain suggests a suboptimal utilization of an available and robust neural coding scheme.

\section{Discussion}

Behavioral and physiological estimates of detection thresholds for AM sounds were made in the same species (rabbit) and compared with human thresholds estimated with matched procedures and stimuli. The results suggest that synchrony information is available in the neural responses that can explain the sensitive psychophysical thresholds of human listeners, whereas rabbit behavioral thresholds are better explained by neural coding based on the less sensitive rate thresholds. This summary of the results is based on straightforward comparisons of the behavioral thresholds to the best neural thresholds. More sophisticated strategies for pooling information across the population of neurons could be applied to the rate-based thresholds (Rosen et al.,
sAM Tone

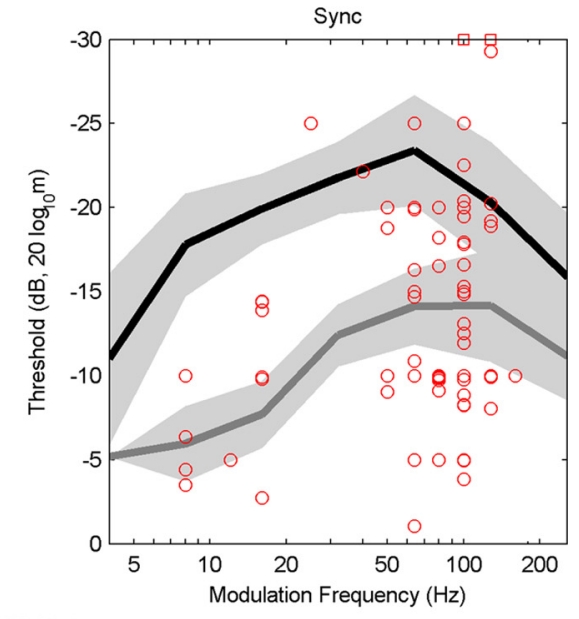

sAM Noise

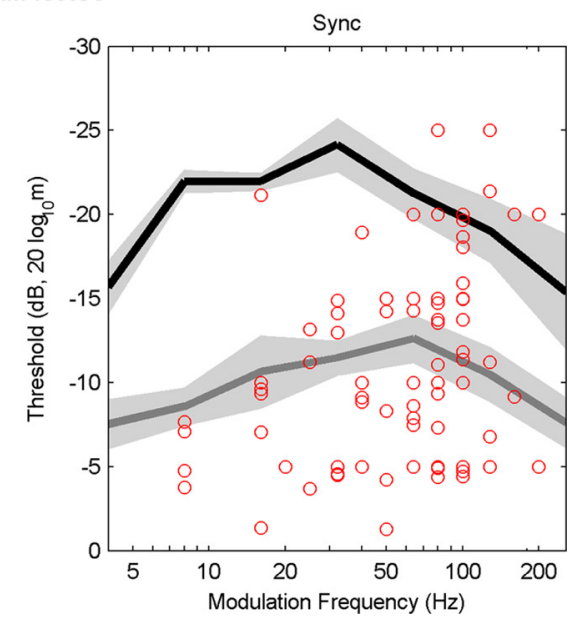

Figure 3. Neural thresholds as a function of modulation frequency. Neural thresholds are shown for responses to sAM tones (a) and SAM nean behavioral thresholds from Figure 1 are shown for human (black line) and rabbit (gray line; mean \pm 1 SD, light gray regions). More sensitive thresholds are at the top of each graph. Left, Blue symbols indicate single neuron thresholds for AM detection based hanges in average discharge rate. Right, Red symbols indicate single neuron thresholds based on synchronization to the sAM stimulus average discharge rate thresholds could explain rabbit, but not human, detection thresholds, whereas synchronization thresholds often matched the human threshold values (see Materials and Methods).

2010), but such strategies cannot explain the large differences in behavioral thresholds between human and rabbit (e.g., thresholds differ by $\sim 15 \mathrm{~dB}$ for sAM tones at low $F_{\mathrm{m}}$; Fig. $3 a$ ). Although other analyses, such as correlations of discharge times across neurons, were not explored here, they would not affect the basic conclusions because simple average rate-based thresholds were adequate to explain the rabbit behavioral thresholds.

The most sensitive rate thresholds are consistent with rabbit behavioral thresholds, whereas the most sensitive synchronybased thresholds are more sensitive than rabbit behavior and are sufficient to explain human behavioral thresholds. A simple interpretation of these results would suggest that humans use temporal information in the neural responses to AM sounds, and rabbits do not. This interpretation, however, assumes that neural responses in humans are similar to those in rabbit, in terms of both rate and temporal information. It is also possible that human psychophysical thresholds are supported by neural rate thresholds, but that rate thresholds are more sensitive in human than in rabbit. However, it is unclear what factors could result in lower rate thresholds in humans. With the exception of sharpness 

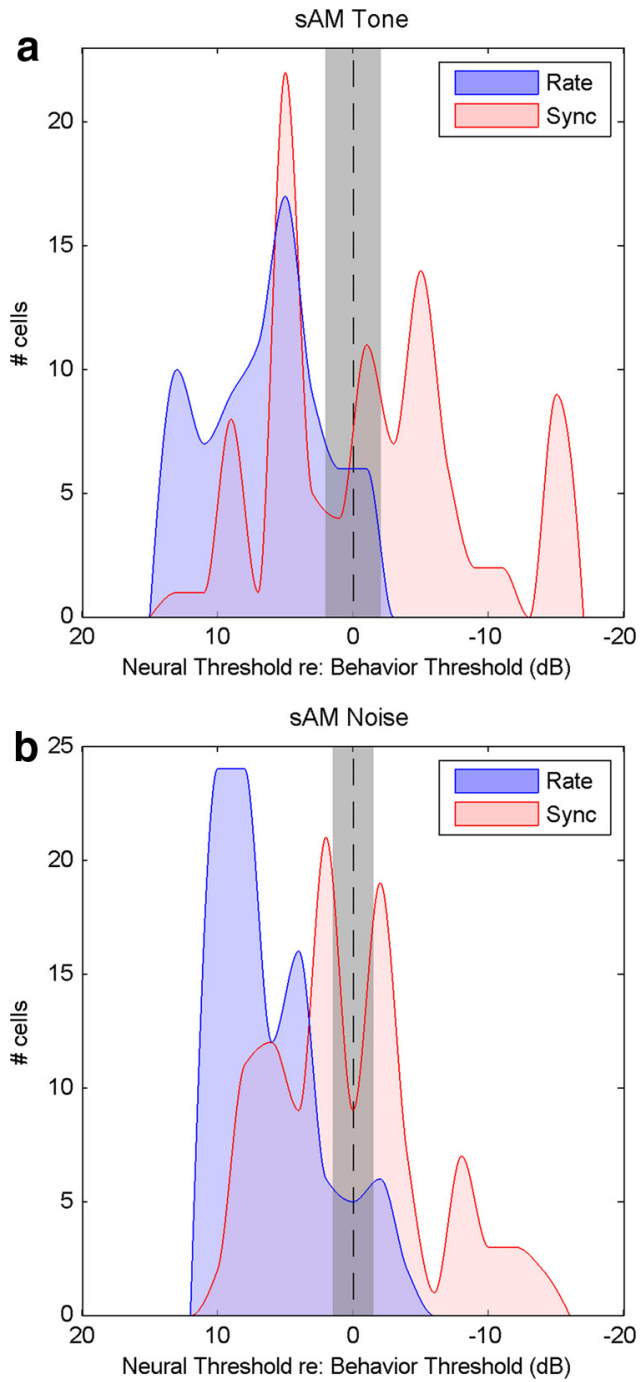

Figure 4. Histograms of neural thresholds combined across modulation frequencies referenced to the mean rabbit behavioral threshold. Mean behavioral threshold for rabbit is shown as the vertical dashed line $\pm 1 S D$ (gray). Thresholds based on average discharge rate are shown in blue; thresholds based on synchronization (sync) to the stimulus envelope are displayed in red. $\boldsymbol{a}$, Distributions of sAM detection thresholds with respect to the mean behavioral threshold for $5 \mathrm{kHz}$ sAM tones. $\boldsymbol{b}$, Distributions of $s$ AM detection thresholds with respect to mean behavioral threshold for sAM noise. For both SAM tone and SAM noise conditions, the most sensitive (right-most) rate thresholds were consistent with mean behavioral thresholds in rabbit, whereas sync thresholds were generally more sensitive than mean behavioral thresholds (i.e., the distribution of sync thresholds extends to the right side of the graph).

of tuning, auditory-nerve response properties are generally equivalent across mammalian species (Shera et al., 2002; Joris et al., 2011); however, peripheral tuning bandwidth would not be a factor for detecting or processing the envelopes of the AM tone stimuli, which have narrowband spectra. AM tuning, which evolves along the ascending auditory pathway, depends largely upon central processing mechanisms (Nelson and Carney, 2004; Davis et al., 2010). It is possible that these central mechanisms are better developed in humans, resulting in lower-rate thresholds. Although difficult to formally rule out, this possibility cannot negate the fact that rabbits do not make effective use of the synchrony information that is present in their neural responses to weakly modulated stimuli.

An alternative explanation is that the rabbits were not sufficiently trained in the psychophysical task to detect AM at modu- lation depths where synchrony rather than rate would have been useful. To avoid this potential confound, animals were trained with both fully modulated AM ( $\sim 7000$ trials total) and with modulation depths held near threshold $\left(d^{\prime} \sim 1\right)$ by two-down, one-up tracks $(\sim 7000$ trials total). Animals were then extensively tested ( $\sim 48,000$ trials total) at modulation depths near threshold using the Bayesian procedure.

Previous behavioral and modeling results (Gai et al., 2007) suggest that rabbits do not use temporal information in another auditory task, the detection of a $500 \mathrm{~Hz}$ tone in wideband noise. In that study, detailed behavioral results for detection in an ensemble of reproducible (frozen) masker waveforms were analyzed for both rabbit and human listeners. A variety of decision variables based on stimulus features were compared with the detection results to identify the features of the stimuli used by each subject for the detection task. Differences in performance across different masker waveforms are highly consistent both within and across subjects for each species. Human listeners use energy, temporal envelope, and temporal fine structure cues (Davidson et al., 2009b). In contrast, the pattern of performance across maskers for rabbits was uncorrelated to that of humans. The rabbit performance for tone-in-noise detection could be partially explained by energy cues, but not by temporal features of the stimuli (Gai et al., 2007).

The results presented here cannot be explained by an inherent, system-level inability of the rabbit brain to access available temporal information because the capacity for processing and using such information does exist in the rabbit auditory system. For example, rabbits are behaviorally sensitive to interaural time differences (ITDs) that are relevant for sound localization (Ebert et al., 2008), and midbrain neurons in the rabbit are sensitive to changes in ITD (Stanford et al., 1992; Kuwada et al., 2006). The limitations of temporal information processing by the rabbit brain may thus be confined to the relatively low-frequency range typically used to modulate AM stimuli. How, or even if, these limitations might be adaptively significant, given that the tested sounds include AM frequencies likely to be encountered naturally by rabbits, is unclear.

The large variation in rabbit AM detection thresholds as a function of modulation frequency for AM tones is consistent with the different roles that timing and rate have been suggested to play in AM coding. Gourévitch and Eggermont (2010) showed that neural timing was most effective for coding AM at lowmodulation frequencies in primary auditory cortex of the anesthetized cat, whereas average rate was sufficient to encode AM at high modulation frequencies. Thus, the inability of rabbits to effectively use low-frequency temporal information in neural responses to AM tones is consistent with their high behavioral thresholds for AM detection at low $F_{\mathrm{m}}$.

The results of this study demonstrate that behavioral thresholds for rabbit are significantly higher than for human, and are better explained by neural rate thresholds than by synchrony thresholds. That is, neural synchrony thresholds were too sensitive to explain rabbit behavior. That information present in rabbit IC neuron responses can explain human auditory performance, but is too sensitive to explain rabbit performance, indicates that the rabbit brain makes suboptimal use of the available temporal information in the neural responses to AM stimuli. Stronger correlations of neural rate thresholds in auditory cortex, compared with synchrony thresholds, to behavioral AM thresholds have also been reported in rhesus macaque (Johnson et al., 2012; Niwa et al., 2012). In the related tasks of detection of frequency modulations in acoustic (Lemus et al., 2009) and vibrot- 
actile (Luna et al., 2005) stimuli, neural rate thresholds rather than synchrony, predicted behavioral thresholds (for review, see Romo et al., 2012). These results suggest important limitations for attempts to correlate neural performance to behavior, and in particular caution against assumptions of optimal use of neural information for sensory encoding.

\section{References}

Attias H, Schreiner CE (1997) Low-order temporal statistics of natural sounds. In: Advances in neural information processing systems, Vol 9, pp 27-33. Cambridge: MIT.

Barlow H (2001) Redundancy reduction revisited. Network 12:241-253. CrossRef Medline

Baumann S, Griffiths TD, Sun L, Petkov CI, Thiele A, Rees A (2011) Orthogonal representation of sound dimensions in the primate midbrain. Nat Neurosci 14:423-425. CrossRef Medline

Bendor D, Wang X (2010) Neural coding of periodicity in marmoset auditory cortex. J Neurophysiol 103:1809-1822. CrossRef Medline

Borst A, Theunissen FE (1999) Information theory and neural coding. Nat Neurosci 2:947-957. CrossRef Medline

Clemens J, Kutzki O, Ronacher B, Schreiber S, Wohlgemuth S (2011) Efficient transformation of an auditory population code in a small sensory system. Proc Natl Acad Sci U S A 108:13812-13817. CrossRef Medline

Davidson SA, Gilkey RH, Colburn HS, Carney LH (2009a) Diotic and dichotic detection with reproducible chimeric stimuli. J Acoust Soc Am 126:1889-1905. CrossRef Medline

Davidson SA, Gilkey RH, Colburn HS, Carney LH (2009b) An evaluation of models for diotic and dichotic detection in reproducible noises. J Acoust Soc Am 126:1906-1925. CrossRef Medline

Davis K, Hancock K, Delgutte B (2010) Computational models of inferior colliculus neurons. In: Computational models of the auditory system, pp 129-176. New York: Springer.

Ebert CS Jr, Blanks DA, Patel MR, Coffey CS, Marshall AF, Fitzpatrick DC (2008) Behavioral sensitivity to interaural time differences in the rabbit. Hear Res 235:134-142. CrossRef Medline

Egan J (1975) Signal detection theory and ROC analysis. New York: Academic.

Elliott TM, Theunissen FE (2009) The modulation transfer function for speech intelligibility. PLoS Comput Biol 5:e1000302. CrossRef Medline

Gai Y, Carney LH, Abrams KS, Idrobo F, Harrison JM, Gilkey RH (2007) Detection of tones in reproducible noise maskers by rabbits and comparison to detection by humans. JARO 8:522-538. CrossRef Medline

Goldberg JM, Brown PB (1969) Response of binaural neurons of dog superior olivary complex to dichotic tonal stimuli: some physiological mechanisms of sound localization. J Neurophysiol 32:613-636. Medline

Gourévitch B, Eggermont JJ (2010) Maximum decoding abilities of temporal patterns and synchronized firings: application to auditory neurons responding to click trains and amplitude modulated white noise. J Comput Neurosci 29:253-277. CrossRef Medline

Green DM (1993) A maximum-likelihood method for estimating thresholds in a yes-no task. J Acoust Soc Am 93:2096-2105. CrossRef Medline

Harper NS, McAlpine D (2004) Optimal neural population coding of an auditory spatial cue. Nature 430:682-686. CrossRef Medline

Heffner H, Masterton R (1980) Hearing in glires: domestic rabbit, cotton rat, feral house mouse, and kangaroo rat. J Acoust Soc Am 68:1584-1599. CrossRef

Johnson JS, Yin P, O'Connor KN, Sutter ML (2012) Ability of primary auditory cortical neurons to detect amplitude modulation with rate and temporal codes: neurometric analysis. J Neurophysiol 107:3325-3341. CrossRef Medline

Jørgensen S, Dau T (2011) Predicting speech intelligibility based on the signal-to-noise envelope power ratio after modulation-frequency selective processing. J Acoust Soc Am 130:1475-1487. CrossRef Medline

Joris PX, Yin TC (1998) Envelope coding in the lateral superior olive: III. Comparison with afferent pathways. J Neurophysiol 79:253-269. Medline

Joris PX, Schreiner CE, Rees A (2004) Neural processing of amplitudemodulated sounds. Physiol Rev 84:541-577. CrossRef Medline

Joris PX, Bergevin C, Kalluri R, Mc Laughlin M, Michelet P, van der Heijden M, Shera CA (2011) Frequency selectivity in old-world monkeys corroborates sharp cochlear tuning in humans. Proc Natl Acad Sci U S A 108:17516-17520. CrossRef Medline

Knill DC, Pouget A (2004) The Bayesian brain: the role of uncertainty in neural coding and computation. Trends Neurosci 27:712-719. CrossRef Medline

Kohlrausch A, Fassel R, Dau T (2000) The influence of carrier level and frequency on modulation and beat-detection thresholds for sinusoidal carriers. J Acoust Soc Am 108:723-734. CrossRef Medline

Kontsevich LL, Tyler CW (1999) Bayesian adaptive estimation of psychometric slope and threshold. Vision Res 39:2729-2737. CrossRef Medline

Krishna BS, Semple MN (2000) Auditory temporal processing: responses to sinusoidally amplitude-modulated tones in the inferior colliculus. J Neurophysiol 84:255-273. Medline

Kuiper N (1960) Tests concerning random points on a circle. Proc Koninkl Neder Akad Wetensch 63:38-47

Kuwada S, Fitzpatrick DC, Batra R, Ostapoff EM (2006) Sensitivity to interaural time differences in the dorsal nucleus of the lateral lemniscus of the unanesthetized rabbit: comparison with other structures. J Neurophysiol 95:1309-1322. CrossRef Medline

Langner G, Schreiner CE (1988) Periodicity coding in the inferior colliculus of the cat: I. Neuronal mechanisms. J Neurophysiol 60:1799-1822. Medline

Laurent G (2002) Olfactory network dynamics and the coding of multidimensional signals. Nat Rev Neurosci 3:884-895. CrossRef Medline

Lemus L, Hernández A, Romo R (2009) Neural codes for perceptual discrimination of acoustic flutter in the primate auditory cortex. Proc Natl Acad Sci U S A 106:9471-9476. CrossRef Medline

Levitt H (1971) Transformed up-down methods in psychoacoustics. J Acoust Soc Am 49:467-477. CrossRef Medline

Liang L, Lu T, Wang X (2002) Neural representations of sinusoidal amplitude and frequency modulations in the primary auditory cortex of awake primates. J Neurophysiol 87:2237-2261. Medline

Lu T, Wang X (2004) Information content of auditory cortical responses to time-varying acoustic stimuli. J Neurophysiol 91:301-313. Medline

Luna R, Hernández A, Brody CD, Romo R (2005) Neural codes for perceptual discrimination in primary somatosensory cortex. Nat Neurosci 8:1210-1219. CrossRef Medline

Malone BJ, Scott BH, Semple MN (2010) Temporal codes for amplitude contrast in auditory cortex. J Neurosci 30:767-784. CrossRef Medline

Mao J, Vosoughi A, Carney LH (2013) Predictions of diotic tone-in-noise detection based on a nonlinear optimal combination of energy, envelope, and fine-structure cues. J Acoust Soc Am 134:396-406. CrossRef Medline

Mardia KV, Jupp PE (2000) Directional statistics. Chinchester, NY: John Wiley.

Müller-Preuss P, Flachskamm C, Bieser A (1994) Neural encoding of amplitude modulation within the auditory midbrain of squirrel monkeys. Hear Res 80:197-208. CrossRef Medline

Nelken I, Rotman Y, Bar Yosef O (1999) Responses of auditory-cortex neurons to structural features of natural sounds. Nature 397:154-157. CrossRef Medline

Nelson PC, Carney LH (2004) A phenomenological model of peripheral and central neural responses to amplitude-modulated tones. J Acoust Soc Am 116:2173-2186. CrossRef Medline

Nelson PC, Carney LH (2007) Neural rate and timing cues for detection and discrimination of amplitude-modulated tones in the awake rabbit inferior colliculus. J Neurophysiol 97:522-539. CrossRef Medline

Niwa M, Johnson JS, O'Connor KN, Sutter ML (2012) Activity related to perceptual judgment and action in primary auditory cortex. J Neurosci 32:3193-3210. CrossRef Medline

Rabang CF, Parthasarathy A, Venkataraman Y, Fisher ZL, Gardner SM, Bartlett EL (2012) A computational model of inferior colliculus responses to amplitude modulated sounds in young and aged rats. Front Neural Circuits 6:77. CrossRef Medline

Richards VM (1992) The detectability of a tone added to narrow bands of equal-energy noise. J Acoust Soc Am 91:3424-3435. CrossRef Medline

Rieke F, Bodnar DA, Bialek W (1995) Naturalistic stimuli increase the rate and efficiency of information transmission by primary auditory afferents. Proc Biol Sci 262:259-265. CrossRef Medline

Romo R, Lemus L, de Lafuente V (2012) Sense, memory, and decisionmaking in the somatosensory cortical network. Curr Opin Neurobiol 22:914-919. CrossRef Medline

Rosen MJ, Semple MN, Sanes DH (2010) Exploiting development to evaluate auditory encoding of amplitude modulation. J Neurosci 30:1550915520. CrossRef Medline 
Rosen S (1992) Temporal information in speech: acoustic, auditory and linguistic aspects. Philos Trans R Soc Lond B Biol Sci 336:367-373. CrossRef Medline

Schwarz DM, Zilany MS, Skevington M, Huang NJ, Flynn BC, Carney LH (2012) Semi-supervised spike sorting using pattern matching and a scaled mahalanobis distance metric. J Neurosci Methods 206:120-131. CrossRef Medline

Shera CA, Guinan JJ Jr, Oxenham AJ (2002) Revised estimates of human cochlear tuning from otoacoustic and behavioral measurements. Proc Natl Acad Sci U S A 99:3318-3323. CrossRef Medline

Sivian LJ, White SD (1933) On minimum audible sound fields. J Acoust Soc Am 4:288-321. CrossRef
Smith EC, Lewicki MS (2006) Efficient auditory coding. Nature 439:978982. CrossRef Medline

Smith ZM, Delgutte B, Oxenham AJ (2002) Chimaeric sounds reveal dichotomies in auditory perception. Nature 416:87-90. CrossRef Medline

Stanford TR, Kuwada S, Batra R (1992) A comparison of the interaural time sensitivity of neurons in the inferior colliculus and thalamus of the unanesthetized rabbit. J Neurosci 12:3200-3216. Medline

van der Heijden M, Kohlrausch A (1995) The role of envelope fluctuations in spectral masking. J Acoust Soc Am 97:1800-1807. CrossRef Medline

Viemeister NF (1979) Temporal modulation transfer functions based upon modulation thresholds. J Acoust Soc Am 66:1364-1380. CrossRef Medline 